Несахарный диабет (НД) является редким синдромом, характеризуемым экскрецией аномально больших объемов разведенной мочи и соответствующим повышением потребления жидкости. При отсутствии лечения пациенты с этим заболеванием ощущают существенные трудности в связи с постоянной необходимостью посещения туалета для мочеиспускания и непрекращающейся жаждой. Десмопрессин - синтетический аналог антидиуретического гормона - уже многие десятилетия является лечением выбора при центральной форме НД. Так же как и при любой другой заместительной терапии гормональными препаратами, чем более точно подобрана доза для конкретного пациента, тем лучше и безопаснее ожидаемый клинический ответ. В данной статье мы представляем случай, демонстрирующий клинические трудности, с которыми пришлось столкнуться при лечении пациентки с центральным НД.

КЛЮЧЕВЫЕ СЛОВА: несахарный диабет, центральный несахарный диабет, десмопрессин, Минирин.

\title{
TREATMENT WITH VARIOUS FORMS OF DESMOPRESSIN IN A PATIENT WITH APPARENTLY NON-IDIOPATHIC DIABETES INSIPIDUS
}

\author{
(C) Pigarova E.A.
}

Endocrinology Research Centre, Moscow, Russia

Diabetes insipidus (DI) is a rare syndrome characterized by an excretion of abnormally large volumes of diluted urine and a corresponding increase in fluid intake. In the absence of treatment, patients with this disease experience significant difficulties due to the constant need to visit the toilet for urination and persistent thirst. Desmopressin, a synthetic analogue of the antidiuretic hormone, has been the treatment of choice for the central form of DI for many decades. Just like with any other hormone replacement therapies, the more accurately the dose is chosen for a particular patient, the better and safer the expected clinical response. In this article, we present a clinical case that demonstrates the clinical difficulties encountered in the treatment of a patient with central DI.

KEYWORDS: diabetes insipidus, central diabetes insipidus, desmopressin, Minirin.

\section{ВВЕДЕНИЕ}

Несахарный диабет (НД) является редким синдромом, характеризуемым экскрецией аномально больших объемов разведенной мочи (полиурия) и соответствующим повышением потребления жидкости (полидипсия). Пациенты без лечения испытывают постоянные трудности в связи с постоянной необходимостью посещения туалета для мочеиспускания и непрекращающейся жаждой. Этот порочный круг продолжается и днем, и ночью, что резко нарушает образ жизни пациентов, делает невозможным полноценный сон.

Десмопрессин - синтетический аналог антидиуретического гормона (АДГ) - уже многие десятилетия является лечением выбора при центральной форме НД. И с момента его появления в арсенале врачей качество жизни пациентов с центральной формой НД значимо улучшилось. При центральном НД десмопрессин применяется как заместительное гормональное лечение для компенсации недостатка выработки эндогенного АДГ. Так же как и при любой другой заместительной терапии гормонами, чем более точно подобрана доза для конкретного пациента, тем лучше и безопаснее ожидаемый клинический ответ.
Представляется случай, демонстрирующий клинические трудности, с которыми пришлось столкнуться при лечении пациентки с центральным НД.

\section{КЛИНИЧЕСКИЙ СЛУЧАЙ}

У пациентки Е. в возрасте 19 лет (1983 г.) на фоне резкого похудения появились полиурия и полидипсия около 6-8 л в сутки. На основании гипоизостенурии в анализе мочи по Зимницкому и отсутствия нарушения углеводного обмена диагностирован НД и назначен препарат адиурекрин с положительным эффектом на состояние водно-электролитного обмена, на фоне коррекции которого также отмечалось восстановление массы тела.

Через год лечения переведена на препарат десмопрессина в виде назальных капель - адиуретин по 2 капли 3 раза в сутки. На фоне терапии адиуретином пациентка чувствовала себя лучше, но продолжительность и предсказуемость длительности действия препарата были неоптимальными, что пациентка связывала с трудностями процесса дозирования капель.

В 1984 г. появились выделения из молочных желез, в анализе крови уровень пролактина составил 8700 мЕд/л (норма 76-564). При проведении КТ голов- 
ного мозга выявлена микроаденома гипофиза. Назначалось в течение 1 года лечение бромокриптином в дозе 2,5 мг 2 раза в сутки.

В 2000 г. пациентка отметила возникновение постоянной зябкости, потребность теплее одеваться в межсезонный период и даже летними ночами. При обследовании получены данные за первичный гипотиреоз в исходе аутоиммунного тиреоидита, начата заместительная терапия левотироксином в дозе 25 мкг в сутки, с некоторым улучшением, но через 6 мес пациентка самостоятельно прием препарата прекратила.

В 2005 г. в связи с прекращением поставок препарата адиурекрин переведена на терапию таблетированным препаратом десмопрессина, Минирином, подобрана доза 0,2 мг 3 раза в сутки.

При обследовании в 2006 г. предъявляла жалобы на эпизоды снижения артериального давления до 90/60 мм рт.ст., сопровождающиеся головной болью, выраженной тошнотой, рвотой.

В общих анализах крови и мочи отклонений не выявлено. Электролитный состав крови (натрий, калий, хлориды, кальций, глюкоза) в норме.

Анализ мочи по Зимницкому: объем проб 310-350970-200-250-150-250-350 мл, относительная плотность проб мочи 1019-1020-1004-1010-1018-1009-10051011; дневной диурез 1830 мл, ночной диурез 1000 мл, суточный диурез - 2830 мл.

В гормональном анализе: ТТГ 3,113 мЕд/л (0,25-3,5), св.Т4 -11,3 пмоль/л (9-20), св.Т3 - 4,5 пмоль/л (2,5-5,5), пролактин - 355 мЕд/л (90-540). Свободный кортизол в суточной моче - 144 ммоль/сут (60-413).

МРТ головного мозга: данных за наличие объемного образования не получено, выявлен гипертензионно-гидроцефальный синдром с асимметрией желудочков мозга (левый боковой желудочек умеренно увеличен в объеме, правый боковой, III и IV желудочки - уменьшены, имеется сужение базальной цистерны, пролабирование оптико-хиазмальной цистерны в полость турецкого седла) и спаечные изменения на уровне опто-хиазмальной цистерны с укорочением ножки гипофиза.

По месту жительства в 2011 г. переведена на терапию десмопрессином в виде назального спрея по 20 мкг утром и 10 мкг вечером. После перевода была сделана попытка снижения дозы препарата до 10 мкг 2 раза в сутки (попытка инициирована с экономической целью), на фоне чего усилилась жажда, появилась полиурия, возросло суточное потребление жидкости.

В период 2012-2015 гг. пациентка чередовала различные препараты десмопрессина (спрей Пресайнекс) Вазомирин, таблетки Минирин), в зависимости от наличия в аптеке. На фоне ранее подобранных доз смена препаратов на компенсации центрального НД не отражалась.

В конце 2015 г. пациентке в поликлинике выдается дженерик десмопрессина в таблетках - Натива. Замена препарата проводилась в эквивалентной дозировке 0,2 мг 3 раза в сутки. На фоне приема этого препарата отмечено резкое ухудшение в виде персистирования жажды, полиурии до 4-4,5 л, головной боли. В связи с более низкой эффективностью доза препарата Натива была увеличена до 0,2 мг 4-5 раз в сутки, на фоне которой исчезла полиурия, но жажда и головная боль не регресси- ровали. После 5 дней приема пациентка самостоятельно перешла на таблетированный препарат десмопрессина Минирин, на фоне приема которого в дозе 0,2 мг 3 раза в день самочувствие и выделение жидкости были полностью восстановлены.

В 2016 г. переведена на терапию препаратом десмопрессина в виде лиофилизированных подъязычных таблеток Минирин МЕЛТ. Переход на новый препарат производился с перерасчетом дозы 120 мкг 3 раза в сутки, нарушения самочувствия при таком переходе отмечено не было.

\section{ОБСУЖДЕНИЕ}

История лечения НД исходит к началу 1900-х гг., когда была впервые обнаружена причинно-следственная связь заболевания с повреждением задней доли гипофиза, а в 1918 г. опубликовано описание голландским доктором Ketil Motzfeldt клинического случая женщины с НД, которая потребляла с едой от 2 до 8 гипофизов крупного рогатого скота, на фоне чего у нее уменьшались симптомы НД, ночное выделение мочи снижалось с 2500 до 300 мл [1].

Препарат адиурекрин, с которого началось лечение описываемой нами пациентки, представлял собой высушенный порошок из гипофизов крупного рогатого скота, но предназначался для интраназальной инсуффляции. Основные проблемы, наблюдавшиеся у пациентов при лечении данным средством, - трудности в подборе дозы, аллергические реакции, при длительном лечении - патологические (воспалительные) изменения слизистой носа.

Синтетический аналог вазопрессина, десмопрессин, был синтезирован чешскими химиками под руководством Милана Заорала в 1967 г. [2]. В сравнении с эндогенным гормоном, десмопрессин имеет значительно большую продолжительность времени полужизни, более выраженное антидиуретическое действие и практически полное отсутствие сосудосуживающей активности, что сразу сделало его препаратом выбора для лечения пациентов с центральной формой НД [3].

Действие десмопрессина прогнозировано у каждого конкретного пациента, но, как ранее было отмечено и для эндогенного вазопрессина, характеризуется значимыми различиями между пациентами и независимо от биодоступности препарата, поскольку проявляется В т.ч. и при внутривенном введении препарата. Такая индивидуальная чувствительность к препарату не может быть предсказана такими клинико-демографическими показателями, как пол, возраст, ИМТ, длительность заболевания, объем выделяемой/потребляемой жидкости [4]. Это делает невозможным унифицированный подход к подбору дозы препарата при начале лечения.

Терапевтическая эквивалентность доз различных фармакологических форм десмопрессина (подъязычные таблетки МЕЛТ, таблетки, назальный спрей, назальные капли, раствор для инъекций) и перерасчет дозы препарата хорошо зарекомендовали себя при необходимости смены препарата пациенту, уже получающему десмопрессин [2, 5, 6] (табл. 1).

Эффективность применения перерасчета доз различных форм десмопрессина можно продемонстрировать 
Таблица 1. Сравнение терапевтической эквивалентности различных фармакологических форм десмопрессина [2]

\begin{tabular}{|c|c|c|c|c|}
\hline $\begin{array}{c}\text { Лиофилизированные подъязычные } \\
\text { таблетки МЕЛТ }\end{array}$ & Таблетки & $\begin{array}{l}\text { Назальный } \\
\text { спрей }\end{array}$ & $\begin{array}{c}\text { Назальные } \\
\text { капли }\end{array}$ & $\begin{array}{c}\text { Раствор } \\
\text { для инъекций }\end{array}$ \\
\hline 60 мКг & $100 \mathrm{мг}$ & 5 мкг & 5 мкг & - \\
\hline 120 мкг & 200 мг & 10 мкг & 10 мкг & Менее 0,5 мкг \\
\hline 240 мкг & $400 \mathrm{мг}$ & 20 мкг & 20 мкг & Менее 1 мкг \\
\hline
\end{tabular}

на примере нашей пациентки - исходно подобранная доза десмопрессина в виде таблеток Минирин в дозе 0,2 мг 3 раза в сутки была взаимозаменяема на 10 мкг 3 раза в сутки десмопрессина спрея и 120 мкг 3 раза в сутки лиофилизированных подъязычных таблеток Минирин МЕЛТ.

Однократный «провал» перерасчета доз при назначении препарата Натива, в контексте общего анамнеза лечения пациентки с легким переходом на различные формы десмопрессина, может быть расценен только как отсутствие биоэквивалентности самого препарата-дженерика. Изменение биоэквалентности может происходить вследствие применения других технических условий производства активного вещества, применения иных неактивных ингредиентов в производстве таблетки, соответственно, таблетки десмопрессина (Натива) могут не являться полностью взаимозаменяемыми по отношению к оригинальным таблеткам десмопрессина, в том числе сублингвальной формы (Минирин Мелт).

Клинический случай нашей пациентки интересен также характером этиологии заболевания, который можно определить только приблизительно, как и у многих пациентов, дебют центрального НД у которых произошел в эру до широкой доступности МРТ. Только технологии МРТ позволяют выявлять аутоиммунные этиологии цен- трального НД, при которых наблюдается увеличение размеров гипофиза гипофиза/ножки в течение 6-24 мес от начала первых симптомов заболевания с дальнейшим, как правило, полным регрессом.

Можно предположить, что причиной центрального НД нашей пациентки был аутоиммунный гипофизит, проявлявшийся признаками аденомы гипофиза на КТ и гиперпролактинемией с полным регрессом этих проявлений в дальнейшем. Примечательно, что предполагаемый аутоиммунный процесс, по данным МРТ в динамике заболевания (через 23 года от его начала), оставил спаечные изменения на уровне опто-хиазмальной цистерны с укорочением ножки гипофиза. Наиболее вероятно, беспокоившие пациентку эпизоды снижения АД, сопровождавшиеся головной болью, тошнотой и рвотой, являются следствием гипертензионно-гидроцефального синдрома.

Таким образом, на физиологическом уровне антидиуретическое действие десмопрессина характеризуется выраженными индивидуальными различиями в фармакодинамическом ответе. Понимание этих индивидуальных различий, внедрение в практику подхода перерасчета эквивалентных доз позволит более точно подбирать дозу пациентам, нуждающимся в антидиуретическом лечении.

\section{СПИСОК ЛИТЕРАТУРЫ | REFERENCES}

1. Motzfeldt K. Diabetes Insipidus. Endocrinology. 1918;2(2):112-121 doi: 10.1210/endo-2-2-112

2. Juul KV, Bircher D, Nørgaard JP. Treatment of central diabetes insipidus. Ferring pharmaceuticals. 2014

3. Oiso Y, Robertson GL, Nørgaard JP, Juul KV. Treatment of Neurohypophyseal Diabetes Insipidus. J. Clin. Endocr. Metab. 2013;98(10):3958-3967. doi: 10.1210/jc.2013-2326.

4. Пигарова Е.А. Центральный несахарный диабет: патогенетические и прогностические аспекты, дифференциальная диагностика. Дис. ... канд. мед. наук. - М; 2009. [Pigarova EA. Tsentral'nyy nesakharnyy diabet: patogeneticheskie i prognosticheskie aspekty, differentsial'naya diagnostika. [Dissertation]. Moscow; 2009. (In Russ.)] Доступно по: http:// medical-diss.com/medicina/tsentralnyy-nesaharnyy-diabet-patogeneticheskie-i-prognosticheskie-aspekty-differentsialnaya-diagnostika

5. Пигарова Е.А. Десмопрессин в диагностике и лечении центрального несахарного диабета // Ожирение и метаболизм. - 2013. T. 10. - №1. - C. 50-51. [Pigarova EA. Desmopressin in the diagnosis and treatment of central diabetes insipidus. Obesity and metabolism. 2013;10(1):50-51. (In Russ.)] doi: 10.14341/2071-8713-5073.

6. Пигарова Е.А., Дзеранова Л.К. Диагностика и лечение центрального несахарного диабета // Ожирение и метаболизм. - 2014. T. 11. - №4. - C. 48-55. [Pigarova EA, Dzeranova LK. Diagnosis and treatment of central diabetes insipidus. Obesity and metabolism. 2014;11(4):48-55. (In Russ.)] doi: 10.14341/omet2014448-55

\section{ИНФОРМАЦИЯ ОБ АВТОРАХ [AUTHORS INFO]}

Пигарова Екатерина Александровна, к.м.н. [Ekaterina A. Pigarova, PhD]; адрес: 117036, ул. Дмитрия Ульянова, 11 [address: 11 Dm. Ulyanova street, 117036 Moscow, Russia]; eLibrary SPIN: 6912-6331; e-mail: kpigarova@gmail.com.

\section{ЦИТИРОВАТЬ:}

Пигарова Е.А.. Особенности лечения различными формами десмопрессина пациентки с неидиопатическим несахарным диабетом. // Ожирение и метаболизм. — 2018. - Т.15. — №. 1 - C.50-52. doi: 10.14341/OMET2018150-52

\section{TO CITE THIS ARTICLE:}

Pigarova EA. Treatment with various forms of desmopressin in a patient with apparently non-idiopathic diabetes insipidus. Obesity and metabolism. 2018;15(1):50-52. doi: 10.14341/OMET2018150-52 